\title{
Residual properties of free products
}

\author{
Federico Berlai*
}

\begin{abstract}
Let $\mathcal{C}$ be a class of groups. We give sufficient conditions ensuring that a free product of residually $\mathcal{C}$ groups is again residually $\mathcal{C}$, and analogous conditions are given for LE- $\mathcal{C}$ groups. As a corollary, we obtain that the class of residually amenable groups and the one of LEA groups are closed under taking free products.

Moreover, we consider the pro- $\mathcal{C}$ topology and we characterize special HNN extensions and amalgamated free products that are residually $\mathcal{C}$, where $\mathcal{C}$ is a suitable class of groups. In this way, we describe special HNN extensions and amalgamated free products that are residually amenable.
\end{abstract}

Key words: root property, residually amenable group, sofic group, free product, pro- $\mathcal{C}$ topology, proamenable topology. 2010 AMS Subject Classification: 20E26, 43A07, 20E06, 20E22, 20E18.

\section{Introduction}

Amenable groups were defined in 1929 by von Neumann in his work on the Banach-Tarski paradox and, since then, the study of amenability has been remarkably fruitful in a variety of branches of mathematics. Recently, weak variants of amenability have attracted considerable interest. Examples are the Haagerup property, also known as Gromov's a-T-menability, and coarse amenability, also termed as Guoliang Yu's Property A. These notions appeared in the study of analytic properties of infinite groups.

The main focus of this paper are other, more algebraic, weak forms of amenability: the notions of residual amenability and of LEA (Locally Embeddable into Amenable) groups. Residual amenability is a common generalization of amenability and of residual finiteness. While the stability, or permanence, properties of both the classes of amenable groups and of residually finite groups are well known, the stability properties of the class of residually amenable groups have not yet been investigated. See, for instance, [26] for corresponding open questions.

In 6] residually amenable and LEA groups appear as fundamental examples of sofic [12, 30] and hyperlinear 22 groups. Thus, on the one hand, it seems reasonable to study the (non-)permanence properties of both the classes of residually amenable and LEA groups, in order to have a better understanding also of the latter classes of sofic and hyperlinear groups.

On the other hand, after more than ten years since sofic groups were defined, the following question is still open: Is there a non-sofic/non-hyperlinear group? There are few examples of groups which are known to be sofic but not residually amenable. Hence, new examples of non-residually amenable groups are of great value for the above question, and one way to produce these new examples is to study the non-permanence properties of the class of residually amenable groups.

On yet another perspective, residually amenable groups have recently been investigated in the context of the theory of $L^{2}$-invariants. In [8] the approximation of $L^{2}$-Betti numbers is studied, and in [19] the connections between residually amenable groups and the $L^{2}$-invariants theory are explained, along with deep conjectures they satisfy, such as the Approximation Conjecture on the $L^{2}$-Betti numbers, and the Determinant Conjecture [19. Conjectures 13.1 and 13.2]. Therefore, the (non-)stability properties of the class of residually amenable groups will also yield new examples in this context.

The first aim of the paper is to show that the class of residually amenable groups is closed under free products. To this end, in Section 2 we consider the classes of residually amenable and residually elementary amenable groups. We show that the classical result of Gruenberg [13, Theorem 4.1] cannot be applied to these classes, that is, amenability and elementary amenability are not root classes (see next section for definitions).

\footnotetext{
*Universität Wien, Fakultät für Mathematik, Oskar-Morgenstern-Platz 1, 1090 Wien, Austria. E-mail address: federico.berlai@univie.ac.at.
} 
In particular, a new method is required to deal with free products of residually amenable and residually elementary amenable groups. This is the focus of Section 3 , where we prove that the classes of residually $\mathcal{C}$ groups are closed under free products, when $\mathcal{C}$ satisfies certain conditions. From this, we deduce that the class of residually amenable groups is indeed closed under free products. After that, we obtain analogous results for free products of LEA groups and LE-C groups.

In Section 4, we consider the pro- $\mathcal{C}$ topology and the pro- $\mathcal{C}$ completion of a group, and we study special HNN extensions and amalgamated free products which are residually $\mathcal{C}$. As a corollary, we characterize particular HNN extensions and amalgamated free products which are residually amenable. Our result is motivated by recent developments in the theory of right-angled Artin groups, and by the analogous fact known for residually finite groups.

\section{$1.1 \quad$ Background}

We start with standard terminology and known facts that are used in this paper.

A class of groups is a family of groups closed under isomorphic images. Let $\mathcal{C}$ be a class of groups. Then a group $G$ is residually $\mathcal{C}$ if for every non-trivial element $g \in G$ there exist a group $C \in \mathcal{C}$ and a surjective homomorphism $\varphi: G \rightarrow C$ such that $\varphi(g) \neq e_{C}$. Moreover, $G$ is fully residually $\mathcal{C}$ if for each finite subset $H \subseteq G \backslash\left\{e_{G}\right\}$ there exist a group $C \in \mathcal{C}$ and a surjective homomorphism $\varphi: G \rightarrow C$ such that $e_{C} \notin \varphi(H)$. If $\mathcal{C}$ is closed under finite direct products, then a group is residually $\mathcal{C}$ if and only if it is fully residually $\mathcal{C}$.

A related notion is the one of local embeddability. Let $G$ and $C$ be two groups and $K \subseteq G$ a finite subset. Then a map $\varphi: G \rightarrow C$ is called a $K$-almost-homomorphism if it satisfies the following conditions:

(a1) $\varphi\left(k_{1} k_{2}\right)=\varphi\left(k_{1}\right) \varphi\left(k_{2}\right)$ for all $k_{1}, k_{2} \in K$;

(a2) $\varphi \uparrow_{K}$ is injective.

Let $\mathcal{C}$ be a class of groups. Then a group $G$ is locally embeddable into the class $\mathcal{C}$ ( $G$ is a LE-C group) if for each finite subset $K \subseteq G$ there exist a group $C \in \mathcal{C}$ and a $K$-almost-homomorphism $\varphi: G \rightarrow C$. For instance, if $\mathcal{C}$ is the class of amenable groups then we get the notion of LEA groups, also known as Gromov's initially subamenable groups [12, page 133]. If $\mathcal{C}$ is the class of finite groups, we obtain LEF groups, see e.g. [29].

If the class $\mathcal{C}$ is closed under finite direct products, then being residually $\mathcal{C}$ implies being LE- $\mathcal{C}$ [6, Corollary 7.1.14]. Moreover, if the class $\mathcal{C}$ is also closed under taking subgroups, then a finitely presented group is residually $\mathcal{C}$ if and only if it is LE-C [6, Corollary 7.1.22].

The study of residual properties began with residual finiteness: in the 1930s Levi proved that free groups are residually finite, and soon after that more proofs appeared, as the one of Hall [14]. Remarkable was the work that Mal'cev started in the 1940s on linear groups and residual finiteness. He proved that finitely generated linear groups are residually finite, and that finitely generated residually finite groups are Hopfian [20. He also showed that a split extension of a finitely generated residually finite group by a residually finite group is residually finite. Recently, this result of Mal'cev concerning split extensions was generalized by Arzhantseva and Gal [1, Theorem 7].

Since the 1960s, the interest in residual properties evolved into the study of residually nilpotent, residually free, fully residually free (also known as limit groups or $\omega$-residually free groups) and residually solvable groups. Residually amenable groups are a common generalization of all these classes.

In 1957 Gruenberg introduced the concept of root property, or root class of groups, namely, a non-trivial class $\mathcal{C}$ such that

1. $\mathcal{C}$ is closed under taking subgroups;

2. $\mathcal{C}$ is closed under taking finite direct products;

3. Gruenberg Condition: for any chain $K \unlhd H \unlhd G$ such that $G / H$ and $H / K$ are in $\mathcal{C}$, there exists $L \unlhd G$ such that $L$ is contained in $K$ and $G / L \in \mathcal{C}$.

Finite groups, finite p-groups and solvable groups are known to satisfy the Gruenberg condition [13, while nilpotent groups do not satisfy it. Using the fact that finite groups form a root class, Gruenberg proved that the free product of residually finite groups is residually finite [13, Theorem 4.1].

Before stating our results, let us fix some notations. If $\mathcal{A}$ and $\mathcal{B}$ are two properties of groups, then a group $G$ is $\mathcal{A}$-by- $\mathcal{B}$ if it admits a normal subgroup $N$ satisfying $\mathcal{A}$ such that the quotient group $G / N$ satisfies $\mathcal{B}$. 
The identity of a group $G$ is denoted by $e_{G}$, or simply by $e$ if the group is clear from the context. The conjugate $\mathrm{gHg}^{-1}$ of a subgroup $H \leq G$ is denoted by $H^{g}$, and the free group on $n$ free generators is denoted by $F_{n}$. If $X$ is a set, $\operatorname{Sym}(X)$ denotes the group of permutations of $X, \operatorname{Sym}_{0}(X)$ the group of permutations of $X$ with finite support and $\operatorname{Sym}_{0}^{+}(X)$ the alternating subgroup of $\operatorname{Sym}_{0}(X)$.

\subsection{Main results}

Our first result is the following generalization of the classical theorem of Gruenberg, who showed that free products of residually finite groups are residually finite [13, Theorem 4.1].

Theorem 1.1. Let $\mathcal{C}$ be a class of groups that contains a root class $\mathcal{R}$. Suppose that

1. $\mathcal{C}$ is closed under finite direct products;

2. every $\mathcal{R}$-by- $\mathcal{C}$ group belongs to $\mathcal{C}$;

3. for every group in $\mathcal{C}$ there is a group in $\mathcal{R}$ of the same cardinality.

Then the class of residually $\mathcal{C}$ groups is closed under free products.

We deduce that the class of residually amenable groups is closed under free products. This answers an open question raised by Schick [26].

Corollary 1.2. The classes of residually amenable groups and of residually elementary amenable groups are closed under free products.

To prove Corollary 1.2 from Theorem 1.1, we use the following result.

Lemma 1.3. If a group is (residually solvable)-by-amenable then it is residually amenable.

If a group is (residually solvable)-by-(elementary amenable) then it is residually elementary amenable.

The next fact is of independent interest. See Example 2.2 for the proof.

Proposition 1.4. The class of residually amenable (resp. residually elementary amenable) groups is not closed under extensions with amenable (resp. elementary amenable) groups.

Our second main result is the following theorem on free products of LE- $\mathcal{C}$ groups.

Theorem 1.5. Let $\mathcal{C}$ be a class of groups, and suppose that

(a) $\mathcal{C}$ is closed under free products

or

(b) $\mathcal{C}$ is closed under finite direct products and the free product of residually $\mathcal{C}$ groups is residually $\mathcal{C}$.

Then the class of LE-C groups is closed under free products.

Corollary 1.6. Let $\mathcal{C}$ be one of the following classes:

1. finite groups;

2. finite p-groups;

3. solvable groups;

4. free groups;

5. elementary amenable groups;

6. amenable groups.

Then the class of LE-C groups is closed under free products.

After recalling the definition of pro- $\mathcal{C}$ topology, we focus our attention on special HNN extensions and doubles of residually amenable groups (see Section 4 for the definitions). 
Theorem 1.7. Let $A$ be a group, $H \leq A$ and consider the special $H N N$ extension $G:=A *_{i d}$ amalgamating the subgroup $H$. Let $\mathcal{C}$ be a class of groups that contains a root class $\mathcal{R}$. Suppose that

1. $\mathcal{C}$ is closed under subgroups and finite direct products;

2. every $\mathcal{R}$-by- $\mathcal{C}$ group belongs to $\mathcal{C}$;

3. for every group in $\mathcal{C}$ there is a group in $\mathcal{R}$ of the same cardinality.

Then $G$ is residually $\mathcal{C}$ if and only if $A$ is residually $\mathcal{C}$ and $H$ is closed in the pro-C topology of $A$.

Corollary 1.8. A special $H N N$ extension $G:=A *_{i d}$ is residually amenable if and only if $A$ is residually amenable and the amalgamated subgroup is closed in the proamenable topology of $A$.

Theorem 1.9. Let $A$ be a group, $H \leq A$ a subgroup and consider the double $G=A *_{H}$ A. Let $\mathcal{C}$ be a class of groups that contains a root class $\mathcal{R}$. Suppose that

1. $\mathcal{C}$ is closed under subgroups and finite direct products;

2. every $\mathcal{R}$-by- $\mathcal{C}$ group belongs to $\mathcal{C}$;

3. for every group in $\mathcal{C}$ there is a group in $\mathcal{R}$ of the same cardinality.

Then $G$ is residually $\mathcal{C}$ if and only if $A$ is residually $\mathcal{C}$ and $H$ is closed in the pro-C topology of $A$.

Corollary 1.10. A double $A *_{H} A$ is residually amenable if and only if $A$ is residually amenable and $H$ is closed in the proamenable topology of $A$.

Finally, we extend results on HNN extensions [23, Lemma 1.2] to residual amenability.

Proposition 1.11. Let $A$ be a group, $H, K$ two subgroups of $A$ and $\varphi: H \rightarrow K$ an isomorphism. Suppose that there exists an automorphism $\alpha: A \rightarrow A$ such that $\alpha \uparrow_{H}=\varphi$. If $A$ is amenable then the HNN extension $A *_{\varphi}$ is residually amenable.

\section{The class of residually amenable groups}

In this section, we recall some properties of the class of amenable and elementary amenable groups, and then we focus on residual amenability. Very basic permanence properties of this class of groups can be obtained using [6, §2.2]: the known stability properties of residually finite groups proved in that book can be instantly extended to the corresponding stability properties of residual amenability. The only result of [6, $\S 2.2]$ that is not immediate to generalize is [6, Lemma 2.2.11], on the residual finiteness of virtually residually finite groups. We prove the analogous fact for residually amenable groups in Lemma 2.3 .

Finite groups and solvable groups are amenable, and the class of discrete amenable groups is closed under taking subgroups, quotients, extensions, and direct limits. If a group contains a copy of a free non-abelian group then it is not amenable, and a virtually amenable groups is amenable. We refer to [6, §2.2] for the proofs of all of these facts.

The class of elementary amenable groups is the smallest class that contains the finite and the solvable groups, and which is closed under the four operations just mentioned, namely, closed under taking subgroups, quotients, extensions, and direct limits. Every elementary amenable group is amenable, but it is now well known that there are many amenable groups which are not elementary amenable, e.g. [11.

The following fact is probably known to specialists, although we have not located an exact reference in the literature.

Proposition 2.1. The group $G:=\prod_{\mathbb{N}} \operatorname{Sym}_{0}(\mathbb{Z})$ contains copies of free non-abelian groups. In particular, $G$ is not amenable.

Proof. The group $\operatorname{Sym}_{0}(\mathbb{Z})$ is elementary amenable because it is locally finite. Let $p_{i}$ be the $(2 i-1)$-th prime number and $q_{i}$ be the $2 i$-th prime number, for all $i \geq 1$. The families $\left\{p_{i}\right\}_{i \geq 1}$ and $\left\{q_{i}\right\}_{i \geq 1}$ are disjoint and their union is exactly the set of all prime numbers. Define, for each $i \geq 1$, the permutations $g_{i}$ and $\gamma_{i}$ of $\mathbb{Z}$ with finite support

$$
g_{i}:=\left(0, p_{i}, 2 p_{i}, \ldots, i p_{i}\right), \quad \gamma_{i}:=\left(0, q_{i}, 2 q_{i}, \ldots, i q_{i}\right)
$$


and consider the elements

$$
g:=\left(g_{n}\right)_{n \in \mathbb{N}}, \quad \gamma:=\left(\gamma_{n}\right)_{n \in \mathbb{N}},
$$

letting $g_{0}=\mathrm{id}_{\mathbb{Z}}=\gamma_{0}$. In particular, the $n$-th components $g_{n}$ and $\gamma_{n}$ of $g$ and $\gamma$ are cycles of length $n+1$. Using the Ping-Pong Lemma we prove that

$$
\langle g, \gamma\rangle \cong\langle g\rangle *\langle\gamma\rangle=F_{2}
$$

Consider the following sets:

$$
\begin{aligned}
& X_{ \pm}:=\left\{w \in G \mid w=g^{ \pm n} w^{\prime}, n \in \mathbb{N} \backslash\{0\}, w^{\prime} \text { is reduced and does not begin with } g^{i}, \quad i \in \mathbb{Z} \backslash\{0\}\right\}, \\
& Y_{ \pm}:=\left\{w \in G \mid w=\gamma^{ \pm n} w^{\prime}, n \in \mathbb{N} \backslash\{0\}, w^{\prime} \text { is reduced and does not begin with } \gamma^{i}, \quad i \in \mathbb{Z} \backslash\{0\}\right\} .
\end{aligned}
$$

These four sets are disjoint and $e_{G}$ does not belong to any of them, because $g$ and $\gamma$ are torsion-free elements of $G$. As

$$
g\left(G \backslash X_{-}\right) \subseteq X_{+}, \quad g^{-1}\left(G \backslash X_{+}\right) \subseteq X_{-}
$$

and

$$
\gamma\left(G \backslash Y_{-}\right) \subseteq Y_{+}, \quad \gamma^{-1}\left(G \backslash Y_{+}\right) \subseteq Y_{-},
$$

we can apply the Ping-Pong Lemma to conclude that $\langle g, \gamma\rangle \cong F_{2}$, so the discrete group $G$ is not amenable.

Thus, there exist amenable groups $G$ such that the power $\prod_{\mathbb{N}} G$ is not amenable. However this cannot occur whenever $G$ is finite as in that case $\prod_{\mathbb{N}} G$ is locally finite.

Example 2.2. Let $A:=\operatorname{Sym}_{0}(\mathbb{Z})$, consider the unrestricted wreath product $G:=A \imath \mathbb{Z}=A^{\mathbb{Z}} \rtimes_{\sigma} \mathbb{Z}$ and let

$$
N=:\left\{\left(a_{i}\right)_{i \in \mathbb{Z}} \in A^{\mathbb{Z}} \mid a_{0}=e_{A}\right\} \unlhd A^{\mathbb{Z}} .
$$

Then

1. the subnormal chain of subgroups $N \unlhd A^{\mathbb{Z}} \unlhd G$ does not satisfy the Gruenberg condition;

2. the group $G$ is sofic but not residually amenable.

The groups $G / A^{\mathbb{Z}} \cong \mathbb{Z}$ and $A^{\mathbb{Z}} / N \cong A$ are both elementary amenable. Suppose now that the Gruenberg condition holds, so that there exists a normal subgroup $L \unlhd G$ such that $L \subseteq N$ and $G / L$ is amenable.

As $L \unlhd G$, we have that

$$
L \subseteq \bigcap_{\substack{g=(e, z) \\ z \in \mathbb{Z}}} N^{g}
$$

Let $g=(e, z)$, then

$$
\begin{aligned}
N^{g} & =\left\{(e, z)(n, 0)(e, z)^{-1} \mid n \in N\right\}=\left\{\left(\sigma_{z}(n), z\right)(e,-z) \mid n \in N\right\} \\
& =\left\{\left(\sigma_{z}(n), 0\right) \mid n \in N\right\}=\left\{\left(a_{i}\right)_{i \in \mathbb{Z}} \in A^{\mathbb{Z}} \mid a_{z}=e_{A}\right\}
\end{aligned}
$$

thus $L$ is contained in

$$
\bigcap_{z \in \mathbb{Z}}\left\{\left(a_{i}\right)_{i \in \mathbb{Z}} \in A^{\mathbb{Z}} \mid a_{z}=e_{A}\right\}=\left\{e_{A^{\mathbb{Z}}}\right\}
$$

that is, $L=\left\{e_{G}\right\}$. This implies that $G / L=G$ is amenable, and this is a contradiction of Proposition 2.1.

Note that $G$ is (residually amenable)-by-amenable, thus it is sofic [6, Proposition 7.5.14]; more precisely it is elementary sofic $[9]$.

Let us prove that $G$ is not residually amenable. Consider the infinite simple group $B:=\operatorname{Sym}_{0}^{+}(\mathbb{Z}) \unlhd A$. The quotient group $A^{\mathbb{Z}} / B^{\mathbb{Z}} \cong \mathbb{Z}_{2}^{\mathbb{Z}}$ is amenable because it is abelian. This implies that $B^{\mathbb{Z}}$ is not amenable, because amenability is closed under extensions and $A^{\mathbb{Z}}$ is not amenable by Proposition 2.1.

The group $B \imath \mathbb{Z}$ is a subgroup of $G=A \imath \mathbb{Z}$, so if $B \imath \mathbb{Z}$ is not residually amenable then neither is $G$. Let $N \unlhd B \imath \mathbb{Z}$ be a normal subgroup with amenable quotient, then the normal subgroup $N \cap B^{\mathbb{Z}} \unlhd B \imath \mathbb{Z}$ is not trivial. Let $e_{G} \neq(n, 0) \in N \cap B^{\mathbb{Z}}$ with $n_{i} \neq e_{B}$ for some $i \in \mathbb{Z}$. The center of $B$ is trivial because $B$ is simple and non-abelian, so there exists $b \in B$ such that $a:=\left[b, n_{i}\right] \neq e_{B}$. 
Consider the new element $\beta \in B^{\mathbb{Z}}$

$$
\beta_{j}= \begin{cases}e_{B} & \text { if } j \neq i, \\ b \neq e_{B} & \text { if } j=i,\end{cases}
$$

then $\alpha:=[n, \beta] \in B^{\mathbb{Z}}$ is an element with only one non-trivial component:

$$
\alpha_{j}= \begin{cases}e_{B} & \text { if } j \neq i, \\ a \neq e_{B} & \text { if } j=i .\end{cases}
$$

An easy computation shows that

$$
(g, z)(\alpha, 0)(g, z)^{-1}=\left(g \sigma_{z}(\alpha) g^{-1}, 0\right) \quad \forall(g, z) \in B \mathfrak{Z},
$$

and these elements are all elements of $N \cap B^{\mathbb{Z}}$, because $N \cap B^{\mathbb{Z}} \unlhd B$ \ $\mathbb{Z}$.

As $e_{B} \neq a \in B$ and $B$ is simple, we have that

$$
\left\{h a h^{-1} \mid h \in B\right\}=B
$$

and that

$$
\begin{aligned}
& B^{\mathbb{Z}} \supseteq N \cap B^{\mathbb{Z}} \supseteq\left\{(g, z)(\alpha, 0)(g, z)^{-1} \mid(g, z) \in B \imath \mathbb{Z}\right\} \\
& =\left\{\left(g \sigma_{z}(\alpha) g^{-1}, 0\right) \mid(g, z) \in B \nmid \mathbb{Z}\right\}=\left\{\sigma_{z}\left(g \alpha g^{-1}\right) \mid(g, z) \in B \nmid \mathbb{Z}\right\}=B^{\mathbb{Z}},
\end{aligned}
$$

that is, $N \cap B^{\mathbb{Z}}=B^{\mathbb{Z}}$.

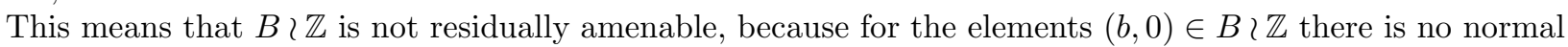
subgroup $N$ with amenable quotient, such that $(b, 0) \notin N$. Hence the group

$$
A \prec \mathbb{Z}=\left(\prod_{z \in \mathbb{Z}} \operatorname{Sym}_{0}(\mathbb{Z})\right) \rtimes \mathbb{Z}
$$

is not residually amenable.

Proposition 1.4 follows from what we just proved.

Lemma 2.3. Let $G$ be a virtually residually amenable group. Then $G$ is residually amenable.

Proof. Let $H \leq G$ be a residually amenable subgroup of finite index of $G$. Then there exists $N \unlhd G$ of finite index in $G$ such that $N \leq H$. Hence $N$ is residually amenable.

Let $g \in G \backslash\{e\}$ be a non-trivial element, we want to find a normal subgroup of $G$ not containing $g$ such that the quotient is amenable. If $g \in G \backslash N$ then it is sufficient to consider the finite quotient $G / N$.

Suppose that $g \in N \backslash\{e\}$. As $N$ is residually amenable, there exists $K \unlhd N$ such that $g \notin K$ and $N / K$ is amenable. Let

$$
G / N=\left\{g_{1} N, g_{2} N, \ldots, g_{r} N\right\}
$$

and define $L:=\bigcap_{i=1}^{r} g_{i} K g_{i}^{-1}$. Then $L \unlhd G, g \notin L$ and $N / L$ has finite index in $G / L$.

We now show that $N / L$ is amenable, that is, $G / L$ is virtually amenable. In fact, as $N \unlhd G$, every conjugate $g_{i} K g_{i}^{-1}$ is again a normal subgroup of $N$, so

$$
N / L \leq\left(N / g_{1} K g_{1}^{-1}\right) \times \cdots \times\left(N / g_{r} K g_{r}^{-1}\right) \cong(N / K)^{r},
$$

Thus $N / L$ is amenable because it is a subgroup of an amenable group.

Hence $G / L$ is indeed virtually amenable, that is, amenable. This concludes the proof, as we have shown that for every non-trivial element $g$ there exists $L \unlhd G$ which does not contain $g$ and with amenable quotient. 


\section{Proof of the main theorems}

We saw in the previous section that amenability is not a root property, thus [13, Theorem 4.1] cannot apply to show that residual amenability is preserved under free products.

In the following lemma we recall some easy properties implied by the Gruenberg condition. The second claim is false in general if the class $\mathcal{C}$ does not satisfy the Gruenberg condition. Proposition 1.4 and Example 2.2 provide a counterexample.

Lemma 3.1. If a class $\mathcal{C}$ satisfies the Gruenberg condition then

1. $\mathcal{C}$ is closed under extensions;

2. a group which is (residually $\mathcal{C})$-by- $\mathcal{C}$ is residually $\mathcal{C}$ [13, Lemma 1.5];

3. free groups are residually $\mathcal{C}$.

Proof. 1. Consider a group $G$ with a normal subgroup $N$ such that $N$ and $G / N$ are in $\mathcal{C}$. Apply the Gruenberg condition to the subnormal chain $\{e\} \unlhd N \unlhd G$ to conclude that $G \in \mathcal{C}$.

2. Let $G$ be a (residually $\mathcal{C}$ )-by- $\mathcal{C}$ group, with a normal residually $\mathcal{C}$ subgroup $N$ such that $G / N \in \mathcal{C}$. Consider an element $g \in G \backslash\left\{e_{G}\right\}$, if $g \notin N$ then the canonical projection $\pi: G \rightarrow G / N$ does not map $g$ to the identity of $G / N$. If $g \in N$ then there exists a surjective homomorphism $\varphi: N \rightarrow C$ such that $C \in \mathcal{C}$ and $\varphi(g) \neq e_{C}$, that is, $g \notin K=\operatorname{ker} \varphi$. As $\varphi$ is surjective, we have that $N / K \cong C \in \mathcal{C}$, so in the subnormal chain $K \unlhd N \unlhd G$ both quotients are groups in $\mathcal{C}$. Thus, applying the Gruenberg condition, we obtain a subgroup $L \unlhd G$ contained in $K$ such that $G / L \in \mathcal{C}$. As $L \subseteq K$, we have that $g \notin L$, and the canonical projection $\psi: G \rightarrow G / L$ does not map $g$ to the identity of $G / L$. This means that $G$ is residually $\mathcal{C}$.

3. Let $G \in \mathcal{C}$ be a non-trivial group, and $g$ be a non-trivial element of $G$. Then either $g$ has infinite order or finite order. In the first case, $\mathcal{C}$ contains all finitely generated torsion-free nilpotent groups. In the second, $\mathcal{C}$ contains all finite $p$-groups, for $p$ a prime dividing the order of $g$.

Free groups are both residually finitely generated torsion-free nilpotent, and residually $p$-finite. Hence, it follows that they are residually $\mathcal{C}$.

In particular, if $\mathcal{C}$ satisfies the Gruenberg condition then $\mathcal{C}$ is closed under finite direct products. This shows that the second condition in the definition of root class is redundant.

Recently, a new characterization of root classes was given.

Theorem 3.2. [27, Theorem 1] Let $\mathcal{C}$ be a class of groups closed under taking subgroups, then the following are equivalent:

1. $\mathcal{C}$ satisfies the Gruenberg condition (that is, $\mathcal{C}$ is a root class);

2. $\mathcal{C}$ is closed under unrestricted wreath products;

3. $\mathcal{C}$ is closed under extensions and for any two groups $G, H \in \mathcal{C}$ the group $\prod_{h \in H} G$ is again in $\mathcal{C}$.

In the following lemma we use the equivalence between the first and the third condition of this characterization.

Lemma 3.3. Let $\mathcal{C}$ be a class of groups that contains a root class $\mathcal{R}$. Suppose that

1. every $\mathcal{R}$-by- $\mathcal{C}$ group belongs to $\mathcal{C}$;

2. for every group in $\mathcal{C}$ there is a group in $\mathcal{R}$ of the same cardinality.

Then a (residually $\mathcal{R})$-by- $\mathcal{C}$ group is residually $\mathcal{C}$.

Proof. Let $G$ be a (residually $\mathcal{R}$ )-by- $\mathcal{C}$ group, with a residually $\mathcal{R}$ normal subgroup $N$ such that $G / N \in \mathcal{C}$.

Consider a non-trivial element $g \in G$. If $g \notin N$ then $g$ is not mapped to the identity of $G / N$ by the canonical projection. If $g \in N \backslash\{e\}$, then there exists a normal subgroup $K \unlhd N$ such that $g \notin K$ and $N / K \in \mathcal{R}$. Let $S$ 
be a set of representatives of $N$ in $G$, then the group $G / N \in \mathcal{C}$ has the same cardinality of $S$. The hypotheses imply that there exists a group $\Gamma \in \mathcal{R}$ of the same cardinality of $G / H$, so

$$
|\Gamma|=|G / N|=|S|
$$

Consider the normal subgroup $L:=\bigcap_{s \in S} K^{s}$ of $G$, then

$$
N / L \hookrightarrow \prod_{s \in S} N / K^{s} \cong \prod_{s \in S} N / K .
$$

The third condition of Theorem 3.2 implies that $\prod_{s \in S} N / K \in \mathcal{R}$, because $|S|=|\Gamma|$ and $\Gamma \in \mathcal{R}$. As $\mathcal{R}$ is closed under taking subgroups, this implies that $N / L \in \mathcal{R}$.

By the Third Isomorphism Theorem for groups

$$
\frac{G / L}{N / L} \cong G / N
$$

so the group $G / L$ is the extension of the group $N / L$, which is in $\mathcal{R}$, by the group $G / N$, which is in $\mathcal{C}$. Thus $G / L \in \mathcal{C}$.

As $L \leq N$ we have that $g \notin L$. Thus, the surjective homomorphism

$$
\varphi: G \rightarrow G / L, \quad \varphi(g):=g L \quad \forall g \in G
$$

maps $g$ to a non-trivial element of $G / L$, and $G$ is residually $\mathcal{C}$.

Lemma 1.3. If a group is (residually solvable)-by-amenable then it is residually amenable.

If a group is (residually solvable)-by-(elementary amenable) then it is residually elementary amenable.

Proof. To apply Lemma 3.3, we note that both the classes of amenable groups and elementary amenable groups are closed under extensions, and they contain the root class of solvable groups. Moreover, for every cardinal number there exist solvable groups of that cardinality, so the hypotheses are satisfied and we can conclude.

In particular, it follows that

Corollary 3.4. If a group is free-by-amenable then it is residually amenable.

Proof. Magnus proved that free groups are residually torsion-free nilpotent [21, hence they are residually solvable. Apply now Corollary 1.3 .

Theorem 1.1. Let $\mathcal{C}$ be a class of groups that contains a root class $\mathcal{R}$. Suppose that

1. $\mathcal{C}$ is closed under finite direct products;

2. every $\mathcal{R}$-by- $\mathcal{C}$ group belongs to $\mathcal{C}$;

3. for every group in $\mathcal{C}$ there is a group in $\mathcal{R}$ of the same cardinality.

Then the class of residually $\mathcal{C}$ groups is closed under free products.

Proof. Let $\left\{G_{i}\right\}_{i \in I}$ be a family of residually $\mathcal{C}$ groups. Without loss of generality we can suppose that $I$ is finite. In fact, if $I$ is not finite, let $w \in *_{i \in I} G_{i}$ be a non-trivial reduced word and let $J \subseteq I$ be the finite set of those indices of $I$ that appear in $w$. Consider then the surjective homomorphism

$$
\psi: *_{i \in I} G_{i} \rightarrow *_{j \in J} G_{j}
$$

such that $\psi\left(g_{j}\right)=g_{j}$ for $j \in J$ and $\psi\left(g_{i}\right)=e$ for $i \in I \backslash J$. Via $\psi$ we see that if the theorem holds when the index set is finite, it will hold in full generality. We prove the theorem for $|I|=2$, as the same argument applies to the other cases.

Moreover, without loss of generality we can suppose that $G_{1}$ and $G_{2}$ are groups in $\mathcal{C}$. In fact, consider a reduced non-trivial word $w \in G_{1} * G_{2}$ containing letters from both groups. Let

$$
A_{i}:=\left\{g \in G_{i} \mid g \text { appears in } w\right\}, \quad \text { for } i=1,2 \text {. }
$$


The sets $A_{i}$ are finite, non-empty subsets of $G_{i}$, and they do not contain the identity of $G_{i}$. The groups $G_{i}$ are residually $\mathcal{C}$, and $\mathcal{C}$ is closed under finite direct products. This means that they are fully residually $\mathcal{C}$, that is, there exist groups $C_{1}, C_{2} \in \mathcal{C}$ and surjective homomorphisms

$$
\varphi_{1}: G_{1} \rightarrow C_{1} \quad \varphi_{2}: G_{2} \rightarrow C_{2}
$$

such that

$$
\varphi_{1}\left(A_{1}\right) \subseteq C_{1} \backslash\left\{e_{G_{1}}\right\}, \quad \varphi_{2}\left(A_{2}\right) \subseteq C_{2} \backslash\left\{e_{G_{2}}\right\}
$$

and that $\varphi_{i} \Upsilon_{A_{i}}$ is injective. Define the surjective homomorphism $\varphi: G_{1} * G_{2} \rightarrow C_{1} * C_{2}$ such that

$$
\varphi \uparrow_{G_{i}}=\varphi_{i} \quad \text { and } \quad \varphi\left(g_{1} \ldots g_{r}\right)=\varphi\left(g_{1}\right) \ldots \varphi\left(g_{r}\right) \quad \forall g_{i} \in G_{\iota_{i}}, \forall r \in \mathbb{N} .
$$

This surjective homomorphism $\varphi$ maps $w$ to a non-trivial element of $C_{1} * C_{2}$, which is a free product of groups belonging to $\mathcal{C}$.

Hence suppose without loss of generality that $G_{1}, G_{2} \in \mathcal{C}$ and consider the canonical projection

$$
\pi: G_{1} * G_{2} \rightarrow G_{1} \times G_{2} .
$$

The class $\mathcal{C}$ is closed under finite direct products, so the image of $\pi$ belongs to $\mathcal{C}$. Let $K=\operatorname{ker} \pi$, then $K$ is free by the Kuroš Subgroup Theorem [25, Theorem 6.3.1], because it has trivial intersection with every $G_{i}$. Free groups are residually $\mathcal{R}$, because $\mathcal{R}$ is a root class, so it follows that the free product $G_{1} * G_{2}$ is (residually $\mathcal{R}$ )-by- $\mathcal{C}$. Apply Lemma 3.3 to conclude that $G_{1} * G_{2}$ is residually $\mathcal{C}$.

Corollary 1.2. The classes of residually amenable groups and of residually elementary amenable groups are closed under free products.

Proof. Let $\mathcal{C}$ be the class of amenable groups, or the one of elementary amenable groups, $\mathcal{R}$ be the class of solvable groups and apply Theorem 1.1 .

Theorem 1.1 guarantees sufficient conditions for a free product of residually $\mathcal{C}$ groups to be again residually $\mathcal{C}$. In general, this is not always the case. A pathological counterexampleis given by the class of abelian groups.

For a non-trivial example when the conclusion of Theorem 1.1 fails, [4, Theorem 6] guarantees that a free product of two non-trivial groups is residually free if and only if the two groups are fully residually free. So consider a residually free group that is not fully residually free, for instance $G=F_{2} \times \mathbb{Z}$. Then $G * G$ is a free product of two residually free groups, and it is not residually free. Note that, in both cases, the two classes considered are not closed under extensions.

In [17] (see also [16, III. 13.]) the following class of groups is considered. Let $\mathcal{C}$ be the smallest class of groups satisfying the following conditions:

$(c 1) \mathcal{C}$ contains all amenable groups;

(c2) if $G, H \in \mathcal{C}$ then $G * H \in \mathcal{C}$;

(c3) if $G$ is virtually $\mathcal{C}$ then $G \in \mathcal{C}$.

It is known that the fundamental group

$$
G_{k}:=\left\langle a_{1}, b_{1}, \ldots, a_{k}, b_{k} \mid \prod_{i=1}^{r}\left[a_{i}, b_{i}\right]\right\rangle
$$

of a closed orientable surface of genus $k \geq 2$ does not belong to $\mathcal{C}$ [17].

Corollary 1.2 and Lemma 2.3 imply that the class of residually amenable groups satisfy the previous conditions $(c 2)$ and $(c 3)$, and condition $(c 1)$ is trivially satisfied. Thus

$$
\mathcal{C} \subsetneq\{\text { residually amenable groups }\}
$$

where the inclusion is strict because the groups given by the presentation (2) are residually finite [], $\S 1.3]$, hence residually amenable. 


\section{Moreover}

$\mathcal{C} \nsubseteq\{$ residually elementary amenable groups $\}$,

in fact this inclusion is not true because there exist finitely generated simple amenable groups [18 that are not elementary amenable (hence, they are not residually elementary amenable). These groups are not elementary amenable because they are infinite and [7, Corollary 2.4] guarantees that a finitely generated simple elementary amenable group is finite.

Also the other inclusion does not hold, that is

\{residually elementary amenable groups $\nsubseteq \nsubseteq \mathcal{C}$,

because the groups given by (2) are residually finite, hence residually elementary amenable.

We now prove Theorem 1.5. Although the class of residually free groups is not closed under taking free products, Theorem 1.5 guarantees that a free product of groups that are locally embeddable into free groups is again locally embeddable into free groups.

Theorem 1.5. Let $\mathcal{C}$ be a class of groups, and suppose that

(a) $\mathcal{C}$ is closed under free products

or

(b) $\mathcal{C}$ is closed under finite direct products and the free product of residually $\mathcal{C}$ groups is residually $\mathcal{C}$.

Then the class of LE-C groups is closed under free products.

Proof. Let $\left\{G_{i}\right\}$ be a family of groups locally embeddable into $\mathcal{C}$. As in the proof of Theorem 1.1, we can suppose that the index set $I$ is finite, and the argument for $|I|=2$ applies to all other cases. Let $K=\left\{w_{1}, \ldots, w_{r}\right\}$ be a finite set of reduced words of $G_{1} * G_{2}$, and consider the finite subsets

$$
K_{i}:=\left\{e_{G_{i}}\right\} \cup\left\{g \in G_{i} \mid g \text { appears in some } w_{i} \text { for } j=1, \ldots, r\right\} \subseteq G_{i}, \quad \text { for } i=1,2 .
$$

The groups $G_{i}$ are locally embeddable into $\mathcal{C}$, hence there exist $C_{i} \in \mathcal{C}$ and $K_{i}$-almost-homomorphisms $\varphi_{i}: G_{i} \rightarrow$ $C_{i}$. Moreover $e_{G_{i}} \in K_{i}$, thus $\varphi_{i}\left(e_{G_{i}}\right)=e_{C_{i}}$. This implies that

$$
\varphi_{i}(g) \neq e_{C_{i}} \quad \forall g \in K_{i} \backslash\left\{e_{G_{i}}\right\} .
$$

Then the map $\varphi: G_{1} * G_{2} \rightarrow C_{1} * C_{2}$ defined by

$$
\varphi \Gamma_{G_{i}}=\varphi_{i} \quad \text { and } \quad \varphi\left(g_{1} \ldots g_{s}\right)=\varphi\left(g_{1}\right) \ldots \varphi\left(g_{s}\right) \quad \forall g_{i} \in G_{\iota_{i}}, \forall s \in \mathbb{N}
$$

is well defined in view of Equation (3). Let us see that $\varphi$ is a $K$-almost-homomorphism.

Consider the reduced words

$$
w=g_{1} \ldots g_{s} \in K, \quad g_{i} \in G_{\iota_{i}} \quad \text { and } \quad w^{\prime}=h_{1} \ldots h_{t} \in K, \quad h_{i} \in G_{\kappa_{i}}
$$

and suppose that $\varphi(w)=\varphi\left(w^{\prime}\right)$. It follows that $s=t$. Moreover, as $w$ and $w^{\prime}$ are reduced words, also $\varphi(w)$ and $\varphi\left(w^{\prime}\right)$ are reduced. This implies that $\iota_{j}=\kappa_{j}$ for all $j=1, \ldots, s$ and that

$$
\varphi_{\iota_{1}}\left(g_{1}\right)=\varphi_{\iota_{1}}\left(h_{1}\right), \quad \ldots, \quad \varphi_{\iota_{s}}\left(g_{s}\right)=\varphi_{\iota_{s}}\left(h_{s}\right) .
$$

As the maps $\varphi_{\iota_{j}}$ are $K_{j}$-almost-homomorphisms and $g_{j}, h_{j} \in K_{\iota_{j}}$, it follows that $g_{j}=h_{j}$ for all $j=1, \ldots, s=t$. This means that $w=w^{\prime}$, that is, $\varphi\left\lceil_{K}\right.$ is injective.

Moreover, condition $(a 1)$ of the definition of $K$-almost-homomorphism is satisfied, because the maps $\varphi_{j}$ satisfy the same conditions for $K_{j}$, respectively. Thus $\varphi$ is a $K$-almost-homomorphism.

If condition $(a)$ holds, then $C_{1} * C_{2} \in \mathcal{C}$ and so $G_{1} * G_{2}$ is locally embeddable into $\mathcal{C}$. On the other hand, if condition $(b)$ holds then $C_{1} * C_{2}$ is residually $\mathcal{C}$. As the class $\mathcal{C}$ is closed under finite direct products, the group $C_{1} * C_{2}$ is fully residually $\mathcal{C}$, that is, there exists a surjective homomorphism $\psi: C_{1} * C_{2} \rightarrow D \in \mathcal{C}$ which is injective on the finite subset $\varphi(K)$ of $C_{1} * C_{2}$. Thus, the composition $\psi \circ \varphi: G_{1} * G_{2} \rightarrow D$ is a $K$-almost-homomorphism, and $G_{1} * G_{2}$ is LE- $\mathcal{C}$.

Conditions $(a)$ and $(b)$ of the previous theorem are independent one from the other. In fact the class of free groups satisfies the first one but not the second, and the class of finite (or amenable, etc.) groups satisfies the second but not the first. As for the residual case, a class of groups $\mathcal{C}$ such that being locally embeddable into $\mathcal{C}$ is not preserved under free products is the class of abelian groups.

Corollary 1.6 follows immediately from Theorem 1.5 . 


\section{The pro- $\mathcal{C}$ topology and applications to HNN extensions and amal- gamated free products}

In this section, we consider the pro- $\mathcal{C}$ topology and the pro- $\mathcal{C}$ completion of a group $G$, we investigate some properties of these constructions for residually $\mathcal{C}$ groups and we give applications to HNN extensions and amalgamated free products.

In 2 the analogous concept of true prosoluble completion for a group is studied, and in 24] pro- $\mathcal{C}$ groups are considered, where $\mathcal{C}$ are various classes of finite groups. We give reference to these works and to the references therein for more about pro- $\mathcal{C}$ completions.

Let $\mathcal{C}$ be a class of groups, define the pro- $\mathcal{C}$ topology of a group $G$ as the topology with the following basis at $e_{G}$

$$
\mathcal{B}:=\{N \unlhd G \mid G / N \in \mathcal{C}\} .
$$

The pro- $\mathcal{C}$ topology of a group is a linear topology, hence it is a group topology, namely, the group operations

$$
\mu: G \times G \rightarrow G, \quad \iota: G \rightarrow G
$$

defined by $\mu(g, h):=g h$ and $\iota(g):=g^{-1}$ are continuous maps, where $G \times G$ is endowed with the product topology.

If $\mathcal{C}$ is closed under subgroups and finite direct products, then the family $\mathcal{B}$ is directed with respect to inclusion: if $N_{1}$ and $N_{2}$ are elements of $\mathcal{B}$ then also $N_{1} \cap N_{2}$ is.

Define hence the pro- $\mathcal{C}$ completion of a group $G$ as the inverse limit

$$
\widehat{G}:=\lim _{N \in \mathcal{B}} G / N
$$

with respect to the canonical surjective homomorphisms $G / N \rightarrow G / M$, for $N \leq M$ in $\mathcal{B}$, and let $\eta$ be the natural homomorphism

$$
\eta: G \rightarrow \widehat{G}, \quad \eta(g):=(g N)_{N \in \mathcal{B}}
$$

We have the following characterizations. Let $G$ be a group and $\mathcal{C}$ be a class closed under subgroups and finite direct products, then the following conditions are equivalent:

(c1) $G \in \mathcal{C}$

(c2) $\left\{e_{G}\right\} \in \mathcal{B}$, that is, the pro- $\mathcal{C}$ topology over $G$ is discrete;

(c3) $\widehat{G}=G$ and $\eta: G \rightarrow G$ is the identity map.

Also the following conditions are equivalent:

$(r c 1) G$ is residually $\mathcal{C}$;

$(r c 2)$ the pro- $\mathcal{C}$ topology over $G$ is Hausdorff;

(rc3) $\eta: G \rightarrow \widehat{G}$ is injective.

For the rest of the section, also if not explicitly stated, $\mathcal{C}$ is assumed being closed under subgroups and finite direct products. The proof of the following lemma carries over from the proof of [15, Theorem 3.1].

Lemma 4.1. Let $G$ be a residually $\mathcal{C}$ group and consider its pro-C topology. A subgroup $H$ contains an open set if and only if it contains some subgroup $N \in \mathcal{B}$.

Moreover, in such a case, $H$ is both open and closed.

We underline the following immediate corollary:

Corollary 4.2. Let $G$ be a residually $\mathcal{C}$ group, $H$ a subgroup and $N \in \mathcal{B}$. Then the subgroup $H N$ is both open and closed in the pro-C topology.

For a subgroup $H \leq G$ the following three equivalent properties characterize to be closed in the pro- $\mathcal{C}$ topology. 
1. for all $g \in G \backslash H$ there exists a surjective homomorphism $\varphi: G \rightarrow C$ such that $C \in \mathcal{C}$ and $\varphi(g) \notin \varphi(H)$;

2. for all $g \in G \backslash H$ there exists $N \in \mathcal{B}$ such that $g \notin H N$;

3. $H=\bigcap\{H N \mid N \in \mathcal{B}\}$.

When $\mathcal{C}=\{$ finite groups $\}$, a subgroup satisfying one of the above conditions is sometimes said to be separable in the profinite topology.

We now turn our attention to HNN extensions and amalgamated free products. Recall that, given a group $A$, two subgroups $H$ and $K$ and $\varphi: H \rightarrow K$ an isomorphism, the HNN extension with base group $A$ and amalgamated subgroups $H$ and $K$ is the group given by the presentation

$$
A *_{\varphi}:=\left\langle A, t \mid t^{-1} h t=\varphi(h) \quad \forall h \in H\right\rangle .
$$

We call the HNN extension special if $H=K$ and $\varphi=\operatorname{id}_{H}$.

Theorem 1.7 extends [28, Theorem 4.2] via Lemma 3.3] to classes of groups $\mathcal{C}$ that satisfy the same hypotheses of that lemma.

Theorem 1.7. Let $A$ be a group, $H \leq A$ and consider the special $H N N$ extension $G:=A *_{i d}$ amalgamating the subgroup $H$. Let $\mathcal{C}$ be a class of groups that contains a root class $\mathcal{R}$. Suppose that

1. $\mathcal{C}$ is closed under subgroups and finite direct products;

2. every $\mathcal{R}$-by- $\mathcal{C}$ group belongs to $\mathcal{C}$;

3. for every group in $\mathcal{C}$ there is a group in $\mathcal{R}$ of the same cardinality.

Then $G$ is residually $\mathcal{C}$ if and only if $A$ is residually $\mathcal{C}$ and $H$ is closed in the pro- $\mathcal{C}$ topology of $A$.

Proof. We first assume that $A \in \mathcal{C}$ and we prove that the HNN extension $G$ is residually $\mathcal{C}$. In this case the pro- $\mathcal{C}$ topology of $A$ is clearly discrete, hence all the subgroups are closed.

Consider the surjective homomorphism $\pi: G \rightarrow A$ defined by $\pi(a)=a$ for all $a \in A$ and $\pi(t)=e_{A}$. This is a homomorphism because the considered HNN extension is special. Let $K:=\operatorname{ker} \pi$, then $A \cap K=\left\{e_{A}\right\}$, and for all $g \in G$ we have that

$$
g^{-1} A g \cap K=g^{-1} A g \cap g^{-1} K g=g^{-1}(A \cap K) g=\left\{e_{A}\right\} .
$$

Hence, $K$ is a free group because it acts freely on the Bass-Serre tree of the HNN extension $A *_{\mathrm{id}}$. This means that $G$ is free-by- $\mathcal{C}$ and in particular (residually $\mathcal{R}$ )-by- $\mathcal{C}$. Lemma 3.3 implies that the group $G$ is residually $\mathcal{C}$.

Suppose that the base group $A$ is residually $\mathcal{C}$ and that $H$ is closed in $A$, we want to show that $G$ is residually $\mathcal{C}$. Let $g$ be a non-trivial element of $G$. By Britton's lemma $g$ has the following reduced form

$$
g=a_{0} t^{\varepsilon_{1}} a_{1} \ldots t^{\varepsilon_{n}} a_{n}
$$

where

- $n$ is minimal, $a_{i} \in A$ and $\varepsilon_{i}= \pm 1$ for all $i=0, \ldots, n$;

- $a_{i} \in(A \backslash H) \cup\{e\}$ for all $i=1, \ldots, n$;

- if $\varepsilon_{i} \varepsilon_{i+1}=-1$ for some $i=1, \ldots, n-1$ then $a_{i} \neq e_{A}$ (and in particular $a_{i} \notin H$ ).

If $g=t^{\varepsilon}$ for some $\varepsilon \in \mathbb{Z} \backslash\{0\}$, then for all normal subgroups $N \unlhd A$ the element $g$ is mapped non-trivially by $\pi: G \rightarrow G_{N}$ to the special HNN extension

$$
G_{N}:=A / N *_{\mathrm{id}}=\left\langle A / N, t \mid t^{-1} x t=x, \forall x \in H N / N\right\rangle,
$$

where id : $H N / N \rightarrow H N / N$ is the identity map. Let $N \in \mathcal{B}$, then $G_{N}$ is residually $\mathcal{C}$ because the base group $A / N$ is in $\mathcal{C}$. Hence there exists a surjective homomorphism $\varphi: G_{N} \rightarrow B$, where $B \in \mathcal{C}$ such that $\varphi\left(t^{\varepsilon}\right) \neq e_{B}$. Thus, the composition of $\varphi \circ \pi$ maps $g$ to a non-trivial element of the group $B \in \mathcal{C}$. 
If $g \neq t^{\varepsilon}$, then in its reduced form given by (4) there are some $a_{i} \neq e_{A}$. As $H$ is closed in $A$ and hence it is separable in the pro- $\mathcal{C}$ topology, there exists a surjective homomorphism $\varphi: A \rightarrow B$, with $B \in \mathcal{C}$, such that for every non-trivial $a_{i}$ (where the elements $a_{i}$ are the elements in the reduced form of $g$ of Equation (4)

$$
\varphi\left(a_{i}\right) \notin \varphi(H)=H N / N,
$$

where $N:=\operatorname{ker} \varphi$. Extend $\varphi: A \rightarrow B$ to $\bar{\varphi}: A *_{\text {id }} \rightarrow B *_{\text {id }}$, where in $B *_{\text {id }}$ the identity map is id: $\varphi(H) \rightarrow \varphi(H)$, that is, the identity map of $H N / N$. The element $\bar{\varphi}(g)$ is non-trivial in $B *_{\text {id }}$, because of the conditions in Equation (5), and $B *_{\mathrm{id}}$ is residually $\mathcal{C}$ by the first part of the theorem, because $B \in \mathcal{C}$.

This means that $G$ is residually (residually $\mathcal{C}$ ), that is, $G$ is residually $\mathcal{C}$.

To conclude, suppose that the special HNN extension $G=A *_{\text {id }}$ is residually $\mathcal{C}$, we want to show that $A$ is residually $\mathcal{C}$ and that the subgroup $H$ is closed in the pro- $\mathcal{C}$ topology of $A$.

As $A \leq G$, it is residually $\mathcal{C}$. Suppose that $H$ is not closed in $A$, so there exists an element $\tilde{a} \in A \backslash H$ such that $\tilde{a} \in \bar{H} N$ for all the normal subgroups $N \in \mathcal{B}$. Consider the non-trivial element $g=t^{-1} \tilde{a} t \tilde{a}^{-1} \in G$. The group $G$ is residually $\mathcal{C}$, so there exists a normal subgroup $K \unlhd G$ such that $g \notin K$ and $G / K \in \mathcal{C}$.

Let $N:=K \cap A \unlhd A$, then $A / N \in \mathcal{C}$, because

$$
A / N=\frac{A}{A \cap K} \cong \frac{A K}{K} \leq G / K
$$

Extend the canonical projection $\pi: A \rightarrow A / N$ to

$$
\bar{\pi}: A *_{\mathrm{id}} \rightarrow(A / N) *_{\mathrm{id}}
$$

where in the second HNN extension the identity map is id: $H N / N \rightarrow H N / N$. As $A / N \in \mathcal{C}$, it follows that $\tilde{a} \in H N$. So there exist $h \in H$ and $n \in N$ such that $\tilde{a}=h n$. Thus

$$
\bar{\pi}(\tilde{a})=\pi(\tilde{a})=\pi(h) \pi(n)=\pi(h) \in \pi(H)=H N / N
$$

and hence

$$
\bar{\pi}(g)=\bar{\pi}\left(t^{-1} \tilde{a} t \tilde{a}^{-1}\right)=\underbrace{t^{-1} \pi(h) t}_{=\pi(h)} \pi\left(h^{-1}\right)=\pi(h) \pi\left(h^{-1}\right)=e .
$$

Thus $g \in \operatorname{ker} \pi_{N} \leq K$ and in particular $g \in K$. This is a contradiction with the initial choice of the normal subgroup $K$, and this contradiction arises from supposing that $H$ is not closed in $A$.

Corollary 4.3. Let $\mathcal{C}$ be a class of groups as in the previous theorem, $A$ a residually $\mathcal{C}$ group and $H \leq A a$ finite subgroup of $A$. Then the special $H N N$ extension $A *_{i d}$ with associated subgroup $H$ is residually $\mathcal{C}$.

The proof of Corollary 1.8 follows from Theorem 1.7 .

Not surprisingly, a similar statement is true for amalgamated free products. Let $H \leq A, K \leq B$ be groups and $\varphi: H \rightarrow K$ an isomorphism. We call the amalgamated free product $A *_{\varphi} B$ a double if $A=B$ and $\varphi=\operatorname{id}_{H}$. In this case, we write $A *_{H} A$ for $A *_{\varphi} A$, and we denote by $\bar{A}$ the right hand side copy of $A$ in the double.

Theorem 1.9. Let $A$ be a group, $H \leq A$ a subgroup and consider the double $G:=A *_{H} A$. Let $\mathcal{C}$ be a class of groups that contains a root class $\mathcal{R}$. Suppose that

1. $\mathcal{C}$ is closed under subgroups and finite direct products;

2. every $\mathcal{R}$-by- $\mathcal{C}$ group belongs to $\mathcal{C}$;

3. for every group in $\mathcal{C}$ there is a group in $\mathcal{R}$ of the same cardinality.

Then $G$ is residually $\mathcal{C}$ if and only if $A$ is residually $\mathcal{C}$ and $H$ is closed in the pro- $\mathcal{C}$ topology of $A$. 
Proof. Suppose first that $A \in \mathcal{C}$. Let id: $\bar{A} \rightarrow A$ be the isomorphism sending $\bar{a}$ to $a$.

By the universal property of amalgamated free products, there exists a unique homomorphism $\varphi: G \rightarrow A$ such that $\varphi \uparrow_{A}=$ id, $\varphi\left\lceil_{\bar{A}}=\right.$ id:

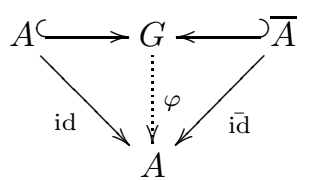

Let $K=\operatorname{ker} \varphi$, then $K$ is generated by the elements $a \bar{a}^{-1}$, for all $a \in A$. Moreover $K \cap A=K \cap \bar{A}=\{e\}$ and hence $K$ is free. The homomorphism $\varphi$ is surjective, so it follows that $G$ is free-by-amenable and thus residually amenable by Corollary 3.4 .

Suppose that $A$ is a residually $\mathcal{C}$ group and that $H \leq A$ is closed in the pro- $\mathcal{C}$ topology of $A$. Let $g \in G$ be a non-trivial element of the double. If $g \in A$ then $\varphi(g)=g \in A \backslash\left\{e_{A}\right\}$. The group $A$ is residually $\mathcal{C}$, so there exists a group $B \in \mathcal{C}$ and a surjective homomorphism $\psi: A \rightarrow B$ such that $\psi(g) \neq e_{B}$. The same argument works for $g \in \bar{A}$.

If $g \notin A \cup \bar{A}$ then

$$
g=a_{1} b_{1} \ldots a_{n} b_{n} \quad a_{i} \in A \backslash H, b_{i} \in \bar{A} \backslash \bar{H} .
$$

The subgroup $H$ is closed in the pro- $\mathcal{C}$ topology of $A$, thus there exist a group $C \in \mathcal{C}$ and a surjective homomorphism $\theta: A \rightarrow C$ such that

$$
\theta\left(a_{i}\right) \notin \theta(H), \quad \bar{\theta}\left(\bar{a}_{i}\right) \notin \bar{\theta}(\bar{H}), \quad \forall i=1, \ldots, n .
$$

Consider the surjective homomorphism

$$
\Theta: G \rightarrow C *_{\theta(H)} C
$$

such that

$$
g \mapsto \Theta(g)=\theta\left(a_{1}\right) \bar{\theta}\left(b_{1}\right) \ldots \theta\left(a_{n}\right) \bar{\theta}\left(b_{n}\right) .
$$

As $\theta\left(a_{i}\right) \notin \theta(H)$ and $\bar{\theta}\left(b_{i}\right) \notin \bar{\theta}(\bar{H})$, it follows that $\Theta(g)$ is not trivial in $C *_{\theta(H)} C$. By the first part of this proof $C *_{\theta(H)} C$ is residually $\mathcal{C}$, hence there exists a quotient $B \in \mathcal{C}$ of $G$ where $g$ is mapped non-trivially.

Suppose now that the amalgamated free product $G$ is residually $\mathcal{C}$ and thus that $A$ is residually $\mathcal{C}$. We need to prove that the amalgamated subgroup $H$ is closed in the pro- $\mathcal{C}$ topology. Suppose it is not, then there exists an element $a \in A \backslash H$ such that $a \in H N$ for all normal subgroups $N \unlhd A$ with $A / N \in \mathcal{C}$. Consider the element $[a, \bar{a}] \in G \backslash(A \cup \bar{A})$.

As the group $G$ is residually $\mathcal{C}$, there exists a normal subgroup $K \unlhd G$ such that $[a, \bar{a}] \notin K$ and $G / K \in \mathcal{C}$. Let $N:=K \cap A$, then $N \unlhd A$ and the quotient

$$
A / N \cong \frac{A K}{K} \leq \frac{G}{K} \in \mathcal{C} .
$$

Thus $a \in H N$, so there exist $h \in H$ and $k \in N$ such that $a=h k$. Moreover $N \unlhd A$, so there exist elements $n, m \in N$ such that $h^{-1} \bar{k}^{-1}=n h^{-1}$ and $k \bar{h}=\bar{h} m$. This means that

$$
\begin{aligned}
{[a, \bar{a}] } & =(h k)^{-1}(\bar{h} \bar{k})^{-1} h k \bar{h} \bar{k}=k^{-1} h^{-1} \bar{k}^{-1} \bar{h}^{-1} h k \bar{h} \bar{k} \\
& =k^{-1}\left(h^{-1} \bar{k}^{-1}\right)(k \bar{h}) \bar{k}=k^{-1}\left(n h^{-1}\right)(\bar{h} m) \bar{k}=k^{-1} n m \bar{k} \in N=K \cap A \leq K .
\end{aligned}
$$

This is a contradiction, because $[a, \bar{a}]$ survives in the quotient $G / K$. Hence the subgroup $H$ is closed in the pro- $\mathcal{C}$ topology of $A$, and the proof is completed.

Corollary 4.4. Let $\mathcal{C}$ be a class of groups as in the previous theorem, $A$ a residually $\mathcal{C}$ group and $H \leq A$ a finite subgroup of $A$. Then the double $A *_{H} A$ is residually $\mathcal{C}$.

Corollary 1.10 follows from Theorem 1.9 
Remark 4.5. Theorem 1.9 is no longer valid if the amalgamated free product is not a double. In fact, in [5] infinite simple groups are constructed as amalgamated free products of free groups (hence, residually finite groups) amalgamating subgroups of finite index (hence, closed in the profinite / proamenable topology of the given free groups).

Thus an amalgamated free product of residually amenable groups, amalgamating two closed subgroups, may be not residually amenable in general.

Consider a solvable group $A$ with two isomorphic subgroups $\varphi: H \rightarrow K$, and suppose that $\varphi$ is the restriction of an automorphism of $A$. It was proved that, in these hypotheses, the HNN extension $A *_{\varphi}$ is residually solvable [23. Lemma 1.2]. Proposition 1.11 is the generalization of this fact just mentioned.

Proposition 1.11. Let $A$ be a group, $H, K$ two subgroups of $A$ and $\varphi: H \rightarrow K$ an isomorphism. Suppose that there exists an automorphism $\alpha: A \rightarrow A$ such that $\alpha \uparrow_{H}=\varphi$. If $A$ is amenable then the HNN extension $A *_{\varphi}$ is residually amenable.

Proof. Consider the two HNN extensions

$$
G=\left\langle A, t \mid t^{-1} h t=\varphi(h) \quad \forall h \in H\right\rangle, \quad G^{\star}=\left\langle A, t \mid t^{-1} a t=\alpha(a) \quad \forall a \in A\right\rangle .
$$

As $H \subseteq A$ and $\alpha \uparrow_{H}=\varphi$, the map $\rho: G \rightarrow G^{\star}$ defined by $\rho(a)=a$ for all $a \in A$ and $\rho(t)=t$ is a well-defined homomorphism. Moreover, $G^{\star}$ is isomorphic to the semidirect product $A \rtimes_{\alpha}\langle t\rangle$ and thus it is amenable-bysolvable, so amenable.

Let $K=\operatorname{ker} \rho$, we have that $A \cap K=\left\{e_{A}\right\}$, thus $K$ intersects trivially each conjugate of $A$ in $G$ because $K$ is normal in $G$. Hence $K$ is a free group, because it acts freely on the Bass-Serre tree of the HNN extension $A *_{\varphi}$. This means that $G$ is free-by-amenable, so it is residually amenable by Corollary 3.4 .

With the same hypotheses of Lemma 1.11, if the base group is residually amenable then the HNN extension need not be residually amenable: Example 2.2 gives the counterexample.

\section{Acknowledgements}

The author is very grateful to his advisor, Goulnara Arzhantseva, for suggesting the topic, for many motivating questions, valuable remarks and corrections on previous versions of this paper. He wants to thank his colleagues of the Geometric Group Theory Research Group at the University of Vienna and Simone Virili for helpful comments and many discussions. Moreover, he thanks Aditi Kar and Nikolay Nikolov for pointing out an error in a previous version of the paper.

The author is supported by the European Research Council (ERC) grant of Prof. Goulnara Arzhantseva, grant agreement no. 259527.

\section{References}

[1] G. N. Arzhantseva, Ś. R. Gal, On approximation properties of semidirect products of groups. ArXiv preprint: http://arxiv.org/abs/1312.7682.

[2] G. N. Arzhantseva, P. de la Harpe, D. Kahrobaei, Z. Šnić, The true prosoluble completion of a group: examples and open problems. With an appendix by Arzhantseva and Šunić. Geom. Dedicata 124 (2007), $5-26$.

[3] G. Baumslag, On generalised free products. Mathematische Zeitschrift, 78 (1962), $423-438$.

[4] B. Baumslag, Residually free groups. Proc. London Math. Soc. (3) 17 (1967), 402 - 418.

[5] M. Burger, S. Mozes, Finitely presented simple groups and products of trees. C. R. Acad. Sci. Paris Sér. I Math. 324 (1997), no. 7, $747-752$.

[6] T. Ceccherini-Silberstein, M. Coornaert, Cellular automata and groups. Springer Monographs in Mathematics. Springer-Verlag, Berlin, 2010. 
[7] C. Chou, Elementary amenable groups. Illinois J. Math. 24 (1980), no. 3, 396 - 407.

[8] B. Clair, Residual amenability and the approximation of $L^{2}$-invariants. Michigan Math. J. 46 (1999), no. 2, 331 - 346 .

[9] Y. de Cornulier, A sofic group away from amenable groups. Math. Ann. 350 (2011), no. 2, 269 - 275.

[10] G. Elek, E. Szabó, On sofic groups. J. Group Theory 9 (2006), no. 2, 161 - 171.

[11] R. I. Grigorchuk, Degrees of growth of finitely generated groups and the theory of invariant means. (Russian) Izv. Akad. Nauk SSSR Ser. Mat. 48 (1984), no. 5, 939 - 985.

[12] M. Gromov, Endomorphisms of symbolic algebraic varieties. J. Eur. Math. Soc. (JEMS) 1 (1999), no. 2, $109-197$.

[13] K. W. Gruenberg, Residual properties of infinite soluble groups. Proc. London Math. Soc. (3) 7 (1957), $29-62$.

[14] M. Hall, Coset representations in free groups. Trans. Amer. Math. Soc., 67 (1949), 421 - 432.

[15] M. Hall, A topology for free groups and related groups. Ann. of Math. (2) 52, (1950). $127-139$.

[16] P. de la Harpe, Topics in geometric group theory. Chicago Lectures in Mathematics. University of Chicago Press, Chicago, IL, 2000.

[17] M. W. Hirsch, W. P. Thurston, Foliated bundles, invariant measures and flat manifolds. Ann. Math. (2) $101(1975), 369-390$.

[18] K. Juschenko, N. Monod, Cantor systems, piecewise translations and simple amenable groups. Ann. of Math. (2) 178 (2013), no. 2, $775-787$.

[19] W. Lück, $L^{2}$-invariants: theory and applications to geometry and K-theory. Ergebnisse der Mathematik und ihrer Grenzgebiete. 3. Folge. A Series of Modern Surveys in Mathematics, 44. Springer-Verlag, Berlin, 2002.

[20] A. I. Mal'cev, On isomorphic matrix representations of infinite groups. (Russian) Mat. Sbornik 8 (50), (1940). 405 - 422; english translation in Amer. Math. Soc. Transl. (2) 45 (1965), 1 - 18.

[21] W. Magnus, Beziehungen zwischen Gruppen und Idealen in einem speziellen Ring. Math. Ann. 111 (1935), no. $1,259-280$.

[22] V. G. Pestov, Hyperlinear and sofic groups: a brief guide. Bull. Symbolic Logic 14 (2008), no. 4, 449 - 480.

[23] E. Raptis, D. Varsos, Residual properties of HNN-extensions with base group an abelian group. Journal of Pure and Applied Algebra 59 (1989), 285 - 290.

[24] L. Ribes, P. Zalesskii, Profinite groups. Second edition. Ergebnisse der Mathematik und ihrer Grenzgebiete. 3. Folge. A Series of Modern Surveys in Mathematics, 40. Springer-Verlag, Berlin, 2010.

[25] D. J. S. Robinson, A course in the theory of groups. Second edition. Graduate Texts in Mathematics, 80 , Springer-Verlag, New York, 1996.

[26] T. Schick, $L^{2}$-determinant class and approximation of $L^{2}$-Betti numbers. Trans. Amer. Math. Soc. 353 (2001), no. 8, $3247-3265$.

[27] E. V. Sokolov, A characterization of root classes of groups. ArXiv preprint: http://arxiv.org/abs/1308.1039.

[28] D. Tieugjo, Root-class residuality of some free constructions. JP J. Algebra Number Theory Appl. 18 (2010), no. 2, $125-143$.

[29] A. M. Vershik, E. I. Gordon, Groups that are locally embeddable in the class of finite groups. (Russian) Algebra i Analiz 9, (1997), no. 1, 71 - 97; english translation in St. Petersburg Math. J. 9 (1998), no. 1, $49-67$. 
[30] B. Weiss, Sofic groups and dynamical systems. Sankhyā Ser. A 62, 3 (2000), 350 - 359. Ergodic theory and harmonic analysis (Mumbai, 1999). 